PFC/JA-90-27

A Stability Criterion for Energetic

Particle-Alfvén Modes

R. Betti and J. P. Freidberg

\author{
August 1990 \\ MIT Plasma Fusion Center \\ Cambridge, MA 02139
}

Submitted for publication in: Physics of Fluids B 


\title{
A stability criterion for energetic particle-alfvén modes
}

\author{
R. Betti and J. P. Freidberg
}

Massachusetts Institute of Technology, Plasma Fusion Center, Cambridge, MA 02139

A stability criterion is derived for energetic particle-Alfvén modes. The criterion is valid for arbitrary aspect ratio, arbitrary $\beta$, noncircular, axisymmetric tori. The plasma is modeled by an MHD core plus a fully kinetic Vlasov species of hot particles. Electron kinetic effects are neglected. In spite of the complexity associated with the analysis of the Vlasov species, a simple but exact stability boundary is derived. The criterion is very fluidlike in nature, suggesting that accurate evaluation in realistic geometries can be accomplished with perhaps only minor modifications to any one of the existing ideal MHD stability codes.

\section{Introduction}

The effect of hot particles on MHD stability has received considerble attention in recent years. Such instabilities are believed to play an important role in existing tokamaks, for instance in the degradation of confinement arising from neutral beam excited $m=1$ internal kinks (i.e. fishbone oscillations). They may also pose an important problem for alpha particle confinement in future ignited tokamaks such as CIT and ITER (i.e. Alfvén gap modes).

The earliest theoretical studies of energetic particle-MHD instabilities involve the EBT configuration where hot electron rings are used to stabilize pressure driven modes. ${ }^{1,2}$ Under certain conditions the hot electrons can create a stabilizing magnetic well while remaining "rigid" against MHD perturbations.

The current theoretical interpretation of the so-called fishbone oscillations is that they are $m=1$ internal kink modes destabilized by trapped hot particles resonant at their magnetic drift frequency. ${ }^{3-6}$ These modes have been observed on many large tokamaks and remain an area of concern regarding the performance of future devices.

Only recently it has been pointed out that highly energetic alpha particles in an ignited tokamak can resonantly interact with the plasma core and destabilize Alfvén modes. Three classes of modes have been identified. Global Alfvén eigenmodes (GAE) are low $n$, 
macroscopic perturbations whose real frequency lies below the lower boundary of the Alfvén continuum. ${ }^{7-13}$ Kinetic Alfvén waves (KAW) are localized modes whose real frequency lies in the Alfvén continuum, and which are discretized by finite Larmor radius effects and damped by a finite parallel electron response. ${ }^{14,15}$ Toroidicity-induced Alfvén eigenmodes (TAE) are low $n$ macroscopic perturbations whose real frequency lies in gaps in Alfvén

continuum arising because of toroidal mode coupling. ${ }^{16,17}$ Under appropriate conditions, each of these modes can be driven unstable by high energy alpha particles.

The instabilities just described have the common feature of a fluid-like MHD mode driven unstable by a high energy kinetic species. The aim of the present paper is to find a general stability criterion for those modes that exist in the stable part of the ideal MHD spectrum, in particular Alfvén waves. We therefore consider a model in which the core plasma is treated as a single MHD fluid and the hot particles as a fully kinetic Vlasov species. By treating the core as a single fluid the effects of electron Landau damping are ignored. Since this is usually a stabilizing influence for the modes under consideration, we obtain a conservative estimate for the stability threshold. The main result of the paper is the derivation of an exact stability criterion valid for a general finite aspect ratio, finite $\beta$, noncircular axisymmetric torus. The simple form of the criterion suggests a powerful means for testing stability against resonant energetic particle driven Alfvén modes in realistic tokamak geometries using existing ideal MHD stability codes.

\section{Model}

Consider a plasma consisting of bulk ions, bulk electrons, and a hot kinetic species such as alpha particles, or neutral beam ions. The electrons are assumed massless and satisfy the following fluid equations

$$
\begin{gathered}
\frac{\partial n_{e}}{\partial t}+\nabla \cdot n_{e} \mathbf{v}_{e}=0 \\
e n_{e}\left(\mathbf{E}+\mathbf{v}_{e} \times \mathbf{B}\right)+\nabla p_{e}=0 \\
p_{e} / n_{e}^{\gamma}=\text { const. }
\end{gathered}
$$


At stated, electron Landau damping is not included in the model. To the extent that the electron-electron collision time is short compared to the characteristic growth time, the neglect of electron Landau damping is justified. In many realistic situations, however, this is not the case. The use of fluid equations for electrons is thus primarily for simplicity, and leads to a conservative stability boundary, since electron Landau damping is usually a stabilizing effect for the class of modes under consideration.

The bulk ions are also modeled by fluid equations as follows

$$
\begin{gathered}
\frac{\partial n_{i}}{\partial t}+\nabla \cdot n_{i} \mathbf{v}_{i}=0 \\
n_{i} m_{i}\left(\frac{\partial \mathbf{v}_{i}}{\partial t}+\mathbf{v}_{i} \cdot \nabla \mathbf{v}_{i}\right)=e n_{i}\left(\mathbf{E}+\mathbf{v}_{i} \times \mathbf{B}\right)-\nabla p_{i} \\
p_{i} / n_{i}^{\gamma}=\text { const. }
\end{gathered}
$$

The ion model neglects Landau damping and finite Larmor radius effects. These are good approximations for many situations of interest satisfying $\omega / k_{\|} v_{t i} \gg 1$ and $\rho_{i} / a \ll 1$. In an additional calculation not presented here, finite Larmor radius effects have been included, and are shown to have virtually no effect on the conclusions deduced from the basic energy relation derived in Section V. Hence for simplicity they are not included in the analysis.

The hot particles are treated as a fully kinetic, collision free species satisfying the Vlasov equation

$$
\frac{\partial f_{\alpha}}{\partial t}+\mathbf{u} \cdot \nabla f_{\alpha}+\frac{Z e}{m_{\alpha}}(\mathbf{E}+\mathbf{u} \times \mathbf{B}) \cdot \nabla_{\mathbf{u}} f_{\alpha}=0 .
$$

This description is "more exact" (and more complicated) than the usual drift kinetic model. Even so, the details of the kinetic theory will not be required to deduce the basic stability criterion.

The model is completed by the addition of the low frequency Maxwell equations

$$
\begin{gathered}
\nabla \times \mathbf{E}=-\frac{\partial \mathbf{B}}{\partial t} \\
\nabla \times \mathbf{B}=\mu_{0} \mathbf{J}=\mu_{0} e\left[n_{i} \mathbf{v}_{i}-n_{e} \mathbf{v}_{e}+Z \int \mathbf{u} f_{\alpha} d \mathbf{u}\right] \\
n_{e}=n_{i}+Z n_{\alpha}
\end{gathered}
$$




$$
\nabla \cdot \mathbf{B}=\mathbf{0}
$$

The overall model can be slightly simplified by introducing the MHD ordering at the outset of the analysis. By assuming $\omega \sim k_{\|} v_{a}$ and $\rho_{i} / a \sim n_{\alpha} / n_{e} \ll 1$, the model reduces to

$$
\begin{gathered}
\frac{\partial \rho}{\partial t}+\nabla \cdot \rho \mathbf{v}=0 \\
\rho \frac{d \mathbf{v}}{d t}=\mathbf{J} \times \mathbf{B}-\nabla p-Z e \int(\mathbf{E}+\mathbf{u} \times \mathbf{B}) f_{\alpha} d \mathbf{u} \\
p / \rho^{\gamma}=\text { const. } \\
\mathbf{E}+\mathbf{v} \times \mathbf{B}=0 \\
\frac{d f_{\alpha}}{d t}=0 \\
\nabla \times \mathbf{E}=-\frac{\partial \mathbf{B}}{\partial t} \\
\nabla \times \mathbf{B}=\mu_{0} \mathbf{J} \\
\nabla \cdot \mathbf{B}=0
\end{gathered}
$$

where $\rho=m_{i} n_{i}, \mathbf{v}=\mathbf{v}_{i}$ and $p=p_{e}+p_{i}$ are the mass density, flow velocity, and particle pressure of the bulk plasma respectively. Note that the momentum equation is an exact consequence of the starting equations. Most of the simplifications arising from the MHD ordering occur in the Ohm's law which reduces to the well known perfect conductivity relation ${ }^{18}$ Also, while $n_{\alpha}$ is assumed small, the hot species pressure $n_{\alpha} T_{\alpha}$ is assumed comparable to the bulk pressure $p$.

The equilibrium and stability analysis that follows is based on the model given by Eqs. (5-12). 


\section{Equilibrium}

The analysis begins with a description of the equilibrium. We consider a general finite aspect ratio, finite $\beta$, noncircular axisymmetric torus. The bulk plasma is assumed at rest (i.e. $\mathbf{v}=0$ ) and most of the current is carried by electrons. The bulk pressure is confined primarily by the $\mathbf{J} \times \mathbf{B}$ force. This is the usual MHD regime.

Focussing on the hot species, we observe that there are several equilibrium forces: electric, magnetic, centrifugal, and thermal. While the Vlasov equation treats them all exactly, in most situations of practical interest the centrifugal and electric field forces are quite small. The hot species is confined by that portion of the $\mathbf{J} \times \mathbf{B}$ force arising from the single fluid $\mathbf{v}_{\alpha} \times \mathbf{B}$ force. In a purely collisionless plasma it is not possible to determine how this force is divided between $\mathbf{v}_{\alpha \phi} \times \mathbf{B}_{p}$ and $\mathbf{v}_{\alpha p} \times \mathbf{B}_{\phi}$ (where $\phi$ and $p$ denote toroidal and poloidal respectively). A transport theory is needed to determine the apportionment. Neoclassical transport theory suggests that poloidal flow is strongly damped by viscosity while toroidal flow can persist for very long times in an axisymmetric geometry. ${ }^{19}$

These arguments indicate that a reasonable choice for the hot species equilibrium distribution function is

$$
\begin{gathered}
f_{\alpha}=f_{\alpha}\left(\epsilon, P_{\phi}\right) \\
\epsilon=\frac{m_{\alpha} u^{2}}{2}+Z e \Phi(R, Z) \\
P_{\phi}=m_{\alpha} R u_{\phi}+Z e \Psi(R, Z) .
\end{gathered}
$$

Here the flux is related to the poloidal field by the usual relation $\mathbf{B}_{p}=\nabla \Psi \times \mathbf{e}_{\phi} / R$. Similarly, $\mathbf{E}=-\nabla \Phi$. In the MHD limit $\mathbf{E}=0$ for static equilibria (from Ohm's law). Thus $\Phi=0$. Since $\epsilon$ and $P_{\phi}$ are exact constants of the motion, Eq. (13) represents an exact solution to the Vlasov equation.

As a further simplification, it is assumed that $f_{\alpha}$ has the form of a rigid rotor

$$
f_{\alpha}=f_{\alpha}\left(\epsilon+\Omega P_{\phi}\right)
$$

where $-\Omega=$ const is the toroidal angular velocity of the hot species. (Note, with this sign convenction $\Omega>0$.) This is an important assumption, critical in the derivation of the 
basic energy relation. The main justifications for rigid rotation are that (1) it produces plausible profiles and (2) it is probably the most stable form of distribution function. Any significant shear in the toroidal flow velocity represents an additional source of free energy, capable of driving new Kelvin-Helmholtz type instabilities and complicating the behavior of existing instabilities. The rigid rotor reduces hot particle plasma instabilities to their most basic form. Note that $f_{\alpha}$ is a general function of $\epsilon+\Omega P_{\phi}$, and not necessarily a Maxwellian.

From Eq. (14) it is straightforward to show that the hot species number density and current density can be written as

$$
\begin{gathered}
n_{\alpha} \equiv \int f_{\alpha} d \mathbf{u}=-\frac{1}{Z e \Omega} \frac{d p_{\alpha}}{d \chi} \\
\mathbf{J}_{\alpha} \equiv Z e \int \mathbf{u} f_{\alpha} d \mathbf{u}=R \frac{d p_{\alpha}}{d \chi} \mathbf{e}_{\phi}
\end{gathered}
$$

Here,

$$
p_{\alpha}(\chi)=\int \frac{m_{\alpha}}{3}\left(\mathbf{u}+\Omega \mathbf{R}_{\phi}\right)^{2} f_{\alpha} d \mathbf{u}
$$

is the hot species particle pressure and

$$
\chi(R, Z)=\psi-\frac{m_{\alpha} \Omega}{2 Z e} R^{2} .
$$

Hereafter, we shall consider $p_{\alpha}(\chi)$, or equivalently $f_{\alpha}\left(\epsilon+\Omega P_{\phi}\right)$ to be a free function.

These relations are substituted into Maxwell's equation. After some simple manipulations we obtain an MHD-like set of equilibrium equations given by

$$
\begin{aligned}
\mathbf{J} \times \mathbf{B}-\nabla\left(p+p_{\alpha}\right)+m_{\alpha} n_{\alpha} \Omega^{2} R \nabla R=0 \\
\nabla \times \mathbf{B}=\mu_{0} \mathbf{J} \\
\nabla \cdot \mathbf{B}=0 .
\end{aligned}
$$

As expected, the hot species enters the momentum balance through an additional pressure gradient force and a centrifugal force. For many practical applications both of these contributions are small compared to the $\nabla p$ term of the bulk plasma. 
Following standard procedures, it is possible to reduce Eq. (19) to a single GradShafranov equation for the flux $\Psi$. This equation has the form

$$
\begin{gathered}
\Delta^{*} \Psi=-F \frac{d F}{d \Psi}-\mu_{0} R^{2}\left(\frac{d p}{d \Psi}+\frac{d p_{\alpha}}{d \chi}\right) \\
\mathbf{B}=\frac{F}{R} \mathbf{e}_{\phi}+\frac{\nabla \Psi \times \mathbf{e}_{\phi}}{R} .
\end{gathered}
$$

In Eq. (20) $F(\Psi), p(\Psi)$ and $p_{\alpha}(\chi)$ are free functions.

This completes the specification of the equilibrium problem. For the stability analysis we shall assume that a solution has been found to Eq. (19) or equivalently Eq. (20).

\section{Stability}

The stability analysis proceeds in a straightforward manner. In an axisymmetric torus all perturbed quantities can be written as $\tilde{Q}(R, Z) \exp (-i \omega t-i n \phi)$. For convenience the analysis is separated into two parts, one involving the bulk plasma and the other the hot species.

A. Bulk plasma analysis. The stability of the bulk plasma is very MHD-like. We introduce the fluid displacement for the bulk plasma

$$
\tilde{\mathbf{v}} \equiv-i \omega \xi
$$

From Eqs. (5)-(11), $\tilde{\mathbf{E}}, \tilde{\mathbf{B}}, \tilde{\mathbf{J}}, \tilde{\rho}$, and $\tilde{p}$ can be easily expressed in terms of $\xi$

$$
\begin{aligned}
\tilde{\mathbf{E}}=i \omega \xi \times \mathbf{B} & \text { Ohm's Law } \\
\tilde{\mathbf{B}}=\nabla \times(\xi \times \mathbf{B}) & \text { Faraday's Law } \\
\tilde{\mathbf{J}}=\left(1 / \mu_{0}\right) \nabla \times \nabla \times(\xi \times \mathbf{B}) & \text { Ampere's Law } \\
\tilde{\rho}=-\nabla \cdot(\rho \xi) & \text { Mass Conservation } \\
\tilde{p}=-\xi \cdot \nabla p-\gamma p \nabla \cdot \xi & \text { Energy Equation }
\end{aligned}
$$

With these substitutions, the momentum equation reduces to

$$
-\omega^{2} \rho \xi=\tilde{\mathbf{J}} \times \mathbf{B}+\mathbf{J} \times \tilde{\mathbf{B}}-\nabla \tilde{p}+\tilde{\mathbf{F}}_{\alpha}
$$


where

$$
\tilde{\mathbf{F}}_{\alpha}=-Z e \int(\tilde{\mathbf{E}}+\mathbf{u} \times \tilde{\mathbf{B}}) f_{\alpha} d \mathbf{u}-Z e \int \mathbf{u} \times \mathbf{B} \tilde{f}_{\alpha} d \mathbf{u} .
$$

The close relationship to ideal MHD is apparent.

B. Hot species analysis. The one remaining quantity to be expressed in terms of $\xi$ is the perturbed distribution function $\tilde{f}_{\alpha}$. Using the well known procedure of integrating back along the unperturbed particle orbits assuming $\operatorname{Im}(\omega)>0$, we find

$$
\tilde{f}_{\alpha}=-\frac{Z e}{m_{\alpha}} \int_{-\infty}^{t}(\tilde{\mathbf{E}}+\mathbf{u} \times \tilde{\mathbf{B}}) \cdot \nabla_{u} f_{\alpha} d t^{\prime} .
$$

For the rigid rotor distribution function

$$
\nabla_{u} f_{\alpha}=\frac{\partial f_{\alpha}}{\partial \epsilon}\left(m_{\alpha} \mathbf{u}+m_{\alpha} \Omega \mathbf{R}_{\phi}\right)
$$

After a short calculation the integrand in Eq. (25) can be written as

$$
(\tilde{\mathbf{E}}+\mathbf{u} \times \tilde{\mathbf{B}}) \cdot \nabla_{u} f_{\alpha}=m_{\alpha} \frac{\partial f_{\alpha}}{\partial \epsilon}\left[\Omega \frac{d}{d t^{\prime}}\left(\xi_{\perp} \cdot \nabla \Psi\right)-i(\omega-n \Omega) \xi_{\perp} \cdot \mathbf{u} \times \mathbf{B}\right] .
$$

This leads to the following expression for the perturbed distribution function

$$
\tilde{f}_{\alpha}=-\frac{\partial f_{\alpha}}{\partial \epsilon}\left[Z e \Omega\left(\xi_{\perp} \cdot \nabla \Psi\right)-i(\omega-n \Omega) \tilde{S}\right]
$$

where

$$
\tilde{S}=Z e \int_{-\infty}^{t} \xi_{\perp} \cdot(\mathbf{u} \times \mathbf{B}) d t^{\prime} .
$$

The first term in Eq. (28) is a simple fluid-like contribution. The second term is quite complicated, requiring an explicit integration along the complex unperturbed orbits.

The final form of the momentum equation is obtained by integrating the fluid-like contributions arising from the hot species. Substituting the first term in Eq. (28) into Eq. (24) yields

$$
Z^{2} e^{2} \Omega\left(\xi_{\perp} \cdot \nabla \Psi\right) \int \mathrm{u} \times \mathbf{B} \frac{\partial f_{\alpha}}{\partial \epsilon} d \mathbf{u}=\frac{d^{2} p_{\alpha}}{d \chi^{2}}\left(\xi_{\perp} \cdot \nabla \Psi\right) \nabla \Psi .
$$

Similarly, the first term in Eq. (24) is evaluated as follows

$$
-Z e \int(\tilde{\mathbf{E}}+\mathbf{u} \times \tilde{\mathbf{B}}) f_{\alpha} d \mathbf{u}=i(\omega-n \Omega) Z e n_{\alpha} \xi_{\perp} \times \mathbf{B}+\frac{d p_{\alpha}}{d \chi} \nabla\left(\xi_{\perp} \cdot \nabla \Psi\right) .
$$


Substituting into the momentum equation [Eq. (23)] gives

$$
-\omega^{2} \rho \xi=\mathbf{F}_{M}(\xi)+\mathbf{F}_{K}(\xi)
$$

Here

$$
\mathbf{F}_{M}(\xi)=\tilde{\mathbf{J}} \times \mathbf{B}+\mathbf{J} \times \tilde{\mathbf{B}}-\nabla\left(\tilde{p}+\tilde{p}_{\alpha}\right)+\tilde{\rho}_{\alpha} \Omega^{2} R \nabla R
$$

with

$$
\begin{gathered}
\tilde{p}_{\alpha}=-\frac{d p_{\alpha}}{d \chi}\left(\xi_{\perp} \cdot \nabla \Psi\right) \\
\tilde{\rho}_{\alpha}=-m_{\alpha} \frac{d n_{\alpha}}{d \chi}\left(\xi_{\perp} \cdot \nabla \Psi\right) .
\end{gathered}
$$

The quantity $\mathbf{F}_{M}(\xi)$ is the analog of the MHD force operator modified by fluid-like contributions to the pressure and centrifugal force arising from the hot species. The quantity $\mathbf{F}_{K}(\xi)$ is a hot species kinetic addition to the force given by

$$
\mathbf{F}_{K}(\xi)=-i(\boldsymbol{\omega}-n \Omega) Z e\left[n_{\alpha} \xi_{\perp} \times \mathbf{B}+\int \mathbf{u} \times \mathbf{B} \frac{\partial f_{\alpha}}{\partial \epsilon} \tilde{S} d \mathbf{u}\right]
$$

The kinetic complications are contained within the trajectory integral $\tilde{S}$.

Equation (32) is the desired form of the momentum equation, expressed entirely in terms of $\xi$.

\section{The General Energy Relation}

Because of the rigid rotor form of the equilibrium distribution function, it is possible to derive a simple and useful energy relation from the basic stability equation [Eq. (32)] by multiplying by $-\xi^{*} / 2$ and integrating over the plasma volume. The left hand side becomes $\omega^{2} K_{M}$ where

$$
K_{M}\left(\xi^{*}, \xi\right)=\frac{1}{2} \int \rho|\xi|^{2} d \mathbf{r}
$$

$K_{M}$ is the familiar MHD kinetic energy normalization.

The first term on the right hand side can be written as

$$
\delta W\left(\xi^{*}, \xi\right)=-\frac{1}{2} \int \xi^{*} \cdot \mathbf{F}_{M}(\xi) d \mathbf{r} .
$$


$\delta W$ is the MHD potential energy contribution modified by the fluid-like hot species contributions. Following well known procedures for mindless MHD algebra, it can be shown that the force operator $\mathbf{F}_{M}(\xi)$ is self-adjoint. One specific self-adjoint form is given by

$$
\begin{gathered}
\delta W=\frac{1}{2} \int d \mathbf{r}\left[\gamma p|\nabla \cdot \xi|^{2}+\frac{\left|\mathrm{B} \cdot \nabla \xi_{\perp}\right|^{2}}{\mu_{0}}+\frac{B^{2}}{\mu_{0}}\left|\nabla \cdot \xi_{\perp}+2 \xi_{\perp} \cdot \kappa\right|^{2}-\frac{4 B^{2}}{\mu_{0}}|\xi \cdot \kappa|^{2}\right. \\
\left.-\frac{m_{\alpha}^{2} \Omega^{2}}{4 Z^{2} e^{2}} \frac{d^{2} p_{\alpha}}{d \chi^{2}}\left|\xi_{\perp} \cdot \nabla R^{2}\right|^{2}+\xi_{\perp}^{*} \xi_{\perp}: \nabla \nabla\left(p+p_{\alpha}+\frac{B^{2}}{2 \mu_{0}}\right)-\frac{\rho_{\alpha} \Omega^{2}}{2} \xi_{\perp}^{*} \xi_{\perp}: \nabla \nabla R^{2}\right]
\end{gathered}
$$

where $\kappa=(\mathbf{B} / B) \cdot \nabla(\mathrm{B} / B)$ is the field line curvature. The self-adjointness property is important because it implies that $\delta W\left(\xi^{*}, \xi\right)$ is real even when $\xi$ is complex. A more intuitive form of the potential energy can be obtained by a different sequence of algebra

$$
\begin{gathered}
\delta W=\frac{1}{2} \int d \mathbf{r}\left\{\frac{\left|\mathbf{Q}_{\perp}\right|^{2}}{\mu_{0}}+\frac{B^{2}}{\mu_{0}}\left|\nabla \cdot \xi_{\perp}+2 \xi_{\perp} \cdot \kappa\right|^{2}+\gamma p|\nabla \cdot \xi|^{2}-\frac{J_{\|}}{B} \xi_{\perp}^{*} \times \mathbf{B} \cdot \mathbf{Q}_{\perp}\right. \\
\left.-\xi_{\perp} \cdot \nabla \psi\left[2\left(\frac{d p}{d \psi}+\frac{d p_{\alpha}}{d \chi}\right) \xi_{\perp}^{*} \cdot \kappa-\Omega^{2} \frac{d \rho_{\alpha}}{d \chi} \xi_{\perp}^{*} \cdot \nabla \frac{R^{2}}{2}\right]\right\}
\end{gathered}
$$

where $\mathbf{Q}_{\perp}=\left[\nabla \times\left(\xi_{\perp} \times \mathbf{B}\right)\right]_{\perp}$. In order, these terms represent line bending, magnetic compression, plasma compression, kink destabilization, curvature destabilization, and centrifugal destabilization.

The last term to evaluate for the energy relation is the kinetic modification denoted by $T_{K}$

$$
T_{K} \equiv-\frac{1}{2} \int \xi^{*} \cdot \mathbf{F}_{K}(\xi) d \mathbf{r}
$$

where $\mathbf{F}_{K}(\xi)$ is given by Eq. (35). The first part of $T_{K}$ can be written as

$$
T_{K}^{(1)}=(\omega-n \Omega) R_{1}
$$

with

$$
R_{1}=\frac{i Z e}{2} \int n_{\alpha} \xi_{\perp}^{*} \cdot\left(\xi_{\perp} \times \mathrm{B}\right) d \mathbf{r}
$$

$R_{1}$ is clearly a real quantity. The second part of $T_{K}$ has the form

$$
T_{K}^{(2)}=\frac{i(\omega-n \Omega) Z e}{2} \int \xi_{\perp}^{*} \cdot(\mathbf{u} \times \mathbf{B}) \frac{\partial f_{\alpha}}{\partial \epsilon} \tilde{S} d \mathbf{u} d \mathbf{r} .
$$


This expression can be simplified by noting that from the definition of $\tilde{S}$

$$
Z e \xi_{\perp}^{*} \cdot(\mathbf{u} \times \mathbf{B})=\frac{d \tilde{S}^{*}}{d t}=i \omega^{*} \tilde{S}^{*}+D \tilde{S}^{*}
$$

where

$$
D \tilde{S}^{*} \equiv\left(\mathbf{u} \cdot \nabla+\frac{Z e}{m_{\alpha}} \mathbf{u} \times \mathbf{B} \cdot \nabla_{\mathbf{u}}\right) \tilde{S}^{*}
$$

$T_{K}^{(2)}$ becomes

$$
T_{K}^{(2)}=-\frac{(\omega-n \Omega)}{2} \int \frac{\partial f_{\alpha}}{\partial \epsilon}\left[\omega^{*}|\tilde{S}|^{2}-i \tilde{S} D \tilde{S}^{*}\right] d \mathbf{u} d \mathbf{r}
$$

The last term in Eq. (46) can be further simplified by writing the complex quantity $\tilde{S}$ as $\tilde{S}=\tilde{A}+i \tilde{C}$. Then,

$$
i \tilde{S} D \tilde{S}^{*}=\frac{i}{2} D\left(\tilde{A}^{2}+\tilde{C}^{2}\right)-(\tilde{C} D \tilde{A}-\tilde{A} D \tilde{C})
$$

Since $D\left(\partial f_{\alpha} / \partial \epsilon\right)=0$ from the equilibrium relations, the contribution to $T_{K}^{(2)}$ from the first term on the right hand side of Eq. (47) is an exact differential which integrates to zero over the phase space volume. Thus, the integration of $i \tilde{S} D \tilde{S}^{*}$ yields a real quantity. $T_{K}^{(2)}$ can now be written as

$$
T_{K}^{(2)}=(\omega-n \Omega)\left[R_{2}-\frac{\omega^{*}}{2} \int \frac{\partial f_{\alpha}}{\partial \epsilon}|\tilde{S}|^{2} d \mathbf{u} d \mathbf{r}\right]
$$

where

$$
R_{2}=\frac{1}{2} \int \frac{\partial f_{\alpha}}{\partial \epsilon}(\tilde{C} D \tilde{A}-\tilde{A} D \tilde{C}) d \mathbf{u} d \mathbf{r}
$$

Combining the contributions from Eqs. (36), (37), (41) and (48), we obtain the following energy relation

$$
\omega^{2} K_{M}=\delta W+(\omega-n \Omega)\left[\bar{R}-\frac{\omega^{*}}{2} \int \frac{\partial f_{\alpha}}{\partial \epsilon}|\tilde{S}|^{2} d \mathbf{u} d \mathbf{r}\right]
$$

and $\bar{R}=R_{1}+R_{2}$ is a real quantity. The final form of the energy relation is obtained by writing $\omega=\omega_{r}+i \omega_{i}$ with $\omega_{i}>0$, and setting the real and imaginary parts of Eq. (50) to zero.

$$
\omega_{i}\left[2 \omega_{r} K_{M}-\bar{R}+\frac{n \Omega}{2} \int \frac{\partial f_{\alpha}}{\partial \epsilon}|\tilde{S}|^{2} d \mathbf{u} d \mathbf{r}\right]=0
$$




$$
\left(\omega_{r}^{2}-\omega_{i}^{2}\right) K_{M}-\delta W-\left(\omega_{r}-n \Omega\right) \bar{R}+\frac{1}{2}\left[\left(\omega_{r}-n \Omega\right) \omega_{r}+\omega_{i}^{2}\right] \int \frac{\partial f_{\alpha}}{\partial \epsilon}|\tilde{S}|^{2} d \mathbf{u} d \mathbf{r}=0 .
$$

Eliminating $\bar{R}$ yields

$$
|\omega-n \Omega|^{2}=\frac{K_{M}}{K_{M}+K_{\alpha}}\left(n^{2} \Omega^{2}-\frac{\delta W}{K_{M}}\right)
$$

where

$$
K_{\alpha}=-\frac{1}{2} \int \frac{\partial f_{\alpha}}{\partial \epsilon}|\tilde{S}|^{2} d \mathbf{u} d \mathbf{r}
$$

Equation (52) is the desired form of the general energy relation.

\section{Discussion}

A. General comments. Several interesting conclusions can be extracted from Eq. (52). For simplicity, consider first the case with no hot kinetic species; that is, an ideal MHD plasma. Equation (52) reduces to

$$
|\omega|^{2}=-\frac{\delta W}{K_{M}} .
$$

If $\delta W>0$ for all allowable perturbations, then Eq. (54) exhibits an obvious contradiction that is only resolved by recognizing that the original assumption $\omega_{i}>0$ must be violated. In other words, when $\delta W>0$, the plasma is stable, consistent with standard MHD analysis.

The next point to note is that if a trial function is found that produces a minimum in $\delta W / K_{M}$ whose value $\left(\delta W / K_{M}\right)_{\min }=0$, then the self-adjointness of $\mathbf{F}_{M}(\xi)$ implies that this trial function is an actual eigenfunction of the system with eigenvalue $\omega_{r}=\omega_{i}=0$. This too is consistent with the well established stability analysis of ideal MHD.

Based on the above information it is tempting to conclude that $\omega=0$ represents the true stability transition. While this is a consequence of the standard analysis, it does not follow from Eq. (54) alone. Specifically, if a trial function is found which makes $\delta W<0$, there is no guarantee that Eq. (54) cannot be satisfied by $\omega_{r} \neq 0, \omega_{i} \leq 0$. Some additional physics is required to establish this conclusion. In ideal MHD this physics is related to the conservation of energy and the Energy Principle. In the case of a hot kinetic species, the additional physics is related to the presence of resonant particles. 
The conclusions for the general case including a hot kinetic species follow in an analogous manner. We consider equilibrium distribution functions satisfying

$$
\frac{\partial f_{\alpha}}{\partial \epsilon}<0
$$

so that $K_{\alpha}>0$. If $\delta W$ (including the hot species fluid-like contributions) is greater than $n^{2} \Omega^{2} K_{M}$ for all allowable trial functions, then Eq. (52) exhibits a contradiction, implying that the assumption $\omega_{i}>0$ is violated; that is

$$
\frac{\delta W}{K_{M}}>n^{2} \Omega^{2}
$$

is a sufficient condition for stability. Note that Eq. (56) implies that there are no unstable modes in the equivalent ideal MHD model without hot particles.

Continuing, for a trial function that minimizes $\delta W / K_{M}$ with $\left(\delta W / K_{M}\right)_{\min }=n^{2} \Omega^{2}$, then the self-adjointness of $\mathbf{F}_{M}(\xi)$ again guarantees that this is a true eigenfunction of the system with eigenvalue $\omega_{r}=n \Omega, \omega_{i}=0$.

Therefore

$$
\left(\frac{\delta W}{K_{M}}\right)_{\min }=n^{2} \Omega^{2}
$$

is a marginal stability point. In a system with resonant particles, the eigenvalues are in general complex, $\omega_{r} \neq 0, \omega_{i} \neq 0$. When $\left(\delta W / K_{M}\right)_{\min }>n^{2} \Omega^{2}$, the contradiction in Eq. (52) implies that $\omega_{i}<0$. When $\left(\delta W / K_{M}\right)_{\min }=n^{2} \Omega^{2}$, we have determined that $\omega_{i}=0$; the eigenvalue is purely real. It is then plausible that when resonant particle effects are included, a trial function which makes $\left(\delta W / K_{M}\right)_{\min }<n^{2} \Omega^{2}$ will lead to $\omega_{i}>0$. We conjecture that modes stable in ideal MHD (e.g. Alfvén waves) become unstable in the presence of a kinetic species when $\left(\delta W / K_{M}\right)_{\min }<n^{2} \Omega^{2}$. This conjecture has been explicitly proven for a related, but slightly simpler system consisting of fluid electrons and fully kinetic ions. ${ }^{20}$ In terms of the standard terminology, the unstable mode has $\omega_{r} \gg \omega_{i}$ with $\omega_{r}^{2} \approx \delta W / K_{M}$ corresponding to a positive energy MHD wave. The stability boundary $\omega_{r}=n \Omega$, represents a transition from positive $\left(\omega_{r}>n \Omega\right)$ to negative $\left(\omega_{r}<n \Omega\right)$ dissipation, the latter giving rise to instability. 
Assuming the validity of this conjecture, then the condition

$$
\frac{\delta W}{K_{M}} \geq n^{2} \Omega^{2}
$$

is sufficient for stability in general and also necessary for Alfvén wave stability.

It is important to recognize that our criterion does not obviously apply to modes which lie in the unstable part of the ideal MHD spectrum, $\left(\delta W / K_{M}\right)_{\min }<0$, such as the $m=1$ internal kink mode with $q(0)<1$. In many cases these modes exist only in the unstable part of the spectrum, never exhibiting a discrete eigenmode with $\omega^{2}>0$ for any set of plasma parameters. Thus our description of the transition from a stable to unstable phase through the critical point $\left(\delta W / K_{M}\right)_{\min }=n^{2} \Omega^{2}>0$ is not applicable. For this reason one cannot recover the energetic-particle kink stability window at high $\beta_{\alpha}^{21,22}$ from the basic energy relation.

Observe that $\delta W$ appearing in Eq. (58) can be larger or smaller than $\delta W$ for pure MHD because of the inclusion of the fluid-like hot particle contributions. In the interesting limit where the hot species $\beta_{\alpha}$ is small compared the bulk $\beta$, these terms are negligible and both forms of $\delta W$ coincide. In this regime, Eq. (58) implies that a hot kinetic species is always destabilizing. An ideal plasma requires $\delta W>0$, whereas the addition of a hot kinetic species, raises the lower limit on $\delta W$ to $\delta W>n^{2} \Omega^{2} K_{M}$.

If finite Larmor radius effects of the bulk ions are introduced, the conclusions remain unchanged. This can be seen heuristically by replacing $\omega^{2}$ with $\omega\left(\omega+\omega_{* i}\right)$ in Eq. (32). Here $\omega_{* i}=\mathbf{k} \cdot \mathbf{V}_{D i}$ is the ion diamagnetic drift frequency. If $\omega_{* i}$ is constant in space, the general stability criterion can be rewritten as $\delta W / K_{M} \geq n^{2} \Omega^{2}+n \Omega \omega_{* i}$. Since $n \Omega \sim \omega_{* \alpha} \gg \omega_{* i}$ the additional term has virtually no effect on the threshold condition.

There is an important practical consequence that follows from Eq. (58). If one is primarily interested in accurate threshold conditions rather than growth rates, then Eq. (58) implies that these conditions can be determined in realistic geometries using pure fluid codes. The complicated particle orbits do not explicitly enter the threshold conditions. For example, in the regime $\beta_{\alpha} \ll \beta$, one can run, with perhaps some modifications, one of the well established ideal MHD stability codes such as PEST, ERATO, or GATO. The 
lowest eigenvalue $\omega_{\min }^{2}$ can be calculated (and may be positive or negative) and then compared to the value of $n^{2} \Omega^{2}$. The threshold condition occurs when $\omega_{\min }^{2}=n^{2} \Omega^{2}$.

B. Applications. The stability criterion given by Eq. (58) can be applied to the problems of the $\mathrm{GAE}^{7-13}$ and $\mathrm{TAE}^{16,17}$ modes. To make comparisons with the existing literature, we must determine the relationship between $\Omega$ and $\omega_{* \alpha}$ (the perpendicular diamagnetic drift frequency). We define $\omega_{* \alpha} \equiv \mathbf{k} \cdot \mathbf{V}_{D}$ where

$$
\begin{gathered}
\mathbf{V}_{D}=\frac{1}{Z e n_{\alpha} B^{2}} \mathbf{B} \times\left(\nabla p_{\alpha}-m_{\alpha} n_{\alpha} R \nabla R\right)=\frac{\mathbf{B} \times \nabla \Psi}{Z e n_{\alpha} B^{2}} \frac{d p_{\alpha}}{d \chi} \\
\mathbf{k}=n \nabla\left[-\phi+\int_{\theta_{0}}^{\theta} \frac{F J}{R^{2}} d \theta^{\prime}\right],
\end{gathered}
$$

$F(\Psi)=R B_{\phi}$, and $\theta$ is an orthogonal poloidal angle. The Jacobian $J$ is related to $B_{p}$ and $\theta$ by the usual relation $J=1 /\left(\mathbf{B}_{p} \cdot \nabla \theta\right)$. Note that $\mathbf{k}$ is defined for any value of $n$ but can only be unambiguously intepreted as the wavenumber for localized modes. A short calculation yields

$$
\omega_{* \alpha}=n \Omega .
$$

Perhaps unexpectly, we see that $\omega_{* \alpha}$, which is proportional to the perpendicular diamagnetic drift velocity, is related to the macroscopic angular velocity $\Omega$ which is predominantly in the parallel (i.e. toroidal) direction. This result is reconciled by observing that the momentum equation for the hot particles implies that the macroscopic fluid velocity and diamagnetic drift velocity are related by $\mathbf{v}_{\alpha}=\mathbf{V}_{D}+\left(v_{\|} / B\right) B$ where $v_{\|}$is a free function. For the rigid rotor distribution function it can be shown that $v_{\|}=-\Omega F(\Psi) / B$. This leads to $\mathrm{v}_{p}=0$ and $v_{\phi}=-\Omega R$.

Consider now Alfvén wave eigenmodes driven unstable by high energy alpha particles. There are two main classes of modes to consider. Global Alfvén eigenmodes ${ }^{7-13}$ (GAE) are low $n$, macroscopic perturbations whose real frequency lies beneath the minimum of the shear Alfvén continuum: $\omega^{2}<\min \left(k_{\|}^{2} v_{a}^{2}\right)$. These modes have been found to be strongly stabilized by toroidicity via coupling to electron Landau damped sideband modes resonating with the shear Alfvén continuum. ${ }^{13}$ Since our model does not include electron 
Landau damping, the GAE would presumably remain unstable in a torus if $\omega_{r}^{2}<\omega_{* \alpha}^{2}$. Even so, in terms of growth rate, the GAE is a much weaker mode than the toroidicityinduced Alfvén eigenmode ${ }^{16,17}$ (TAE) which is the second class of modes to consider.

The TAE mode is a low $n$ macroscopic perturbation whose real frequency lies in a toroidally induced gap in the Alfvén continuum. In the absence of electron Landau damping, (which is often not the dominant stabilizing effect), Van Dam et $\mathrm{al}^{20}$ have shown that in a large but finite aspect ratio circular tokamak, a sufficiently sharp alpha pressure gradient is required for instability: $\omega_{* \alpha}>K \omega_{A}$ where $\omega_{A}=k_{\|}\left(r_{0}\right) v_{a}\left(r_{0}\right)$ and $r_{0}$ is the radius corresponding to the gap. The constant $K$ is of order unity and depends upon the choice of the equilibrium alpha distribution function. For a Maxwellian and a slowing down distribution $K=1$ and $K=1 / 2$ respectively. This threshold is in general agreement with our basic stability criterion if we note that $\omega_{A}^{2}=\delta W / K_{M}$ for the gap mode. However, our criterion predicts $K=1$ for all distribution functions. The discrepancy is due to different definitions of $\omega_{* \alpha}$. In our criterion the critical $\omega_{* \alpha}$ is always given by $\omega_{* \alpha}^{2}=\delta W / K_{M}$. However, the value of $n \Omega=\omega_{* \alpha}$ varies as the alpha particle distribution function changes while holding certain macroscopic quantities fixed (e.g. total number of alphas, total alpha energy, width of the alpha profile, etc.).

Van Dam et al show that the TAE mode may be of serious concern to the ITER project. Their initial calculations predict that ITER will be stable for a slowing down distribution. However, the safety margin is quite small. Other distribution functions, as well as finite aspect ratio, finite $\beta$, and noncircularity could quantitatively change the threshold. This suggests that an accurate threshold calculation, based on Eq. (58) and the procedure described immediately thereafter would indeed be a worthwhile endeavor.

\section{Acknowledgments}

The authors would like to thank Dr. D. J. Sigmar for pointing out the importance of energetic particle-Alfvén modes to future large tokamaks and for several extremely useful discussions during the course of the work. 
This work was supported by the US Department of Energy under contract DE-AC0278ET-51013.

\section{References}

${ }^{1}$ R. A. Dandl, H. O. Eason, G. E. Guest, C. L. Hedrick, H. Ikegami and D. B. Nelson in Plasma Physics and Controlled Nuclear Fusion Research 1974, Proceedings of the 5th International Conference, Tokyo (IAEA, Vienna, 1975) Vol. 2, p. 141.

${ }^{2}$ G. B. Guest, C. L. Hedrick and D. B. Nelson, Phys. Fluids 18, 871 (1975).

${ }^{3}$ L. Chen, R. B. White and M. N. Rosenbluth, Phys. Rev. Lett. 52, 1122 (1984).

${ }^{4}$ B. Coppi and F. Porcelli, Phys. Rev. Lett. 57, 2272 (1986).

${ }^{5}$ B. Coppi. S. Migliuolo and F. Porcelli, Phys. Fluids 31, 1630 (1988).

${ }^{6}$ R. B. White, F. Romanelli and M. N. Bussac, Phys. Fluids B2, 745 (1990).

${ }^{7}$ K. Appert, R. Gruber, F. Troyon and J. Vaclavik, Plasma Phys. 24, 1147 (1982).

${ }^{8}$ D. W. Ross, G. L. Chen and S. M. Mahajan, Phys. Fluids 25, 652 (1982).

${ }^{9}$ S. M. Mahajan, D. W. Ross and G. L. Chen, Phys. Fluids 26, 2195 (1983).

${ }^{10}$ S. M. Mahajan, Phys. Fluids 27, 2238 (1984).

${ }^{11}$ B. Hoffman, Ch. Hollenstein, B. Joye, R. Keller, A. Lietti, J. B. Lister, J.-M. Moret, S. Nowak, J. O'Rourke, A. Pochelon and W. Simm, Plasma Phys. Controlled Fusion 26, 173 (1984).

${ }^{12}$ Y. M. Li, S. M. Mahajan and D. W. Ross, Phys. Fluids 30, 1466 (1987).

${ }^{13}$ G. Y. Fu and J. W. Van Dam, Phys. Fluids B1, 2404 (1989).

${ }^{14}$ M. N. Rosenbluth and P. H. Rutherford, Phys. Rev. Lett. 34, 1428 (1975).

${ }^{15}$ K. T. Tsang, D. J. Sigmar and J. C. Whitson, Phys. Fluids 24, 1508 (1981).

${ }^{16}$ C. Z. Cheng and M. S. Chance, Phys. Fluids 29, 3659 (1986).

${ }^{17}$ G. Y. Fu and J. W. Van Dam, Phys. Fluids B1, 1949 (1989). 
${ }^{18}$ S. I. Braginskii in Reviews of Plasma Physics, edited by M. A. Leontovich, Consultants Bureau, New York, Vol. 1 (1965).

${ }^{19}$ S. P. Hirshman and D. J. Sigmar, Nucl. Fusion 21, 1079 (1981).

${ }^{20}$ C. E. Seyler and J. P. Freidberg, Phys. Fluids 23, 331 (1980).

${ }^{21}$ Y. Z. Zhang, H. L. Berk and S. M. Mahajan, Nucl. Fusion 29, 848 (1989).

${ }^{22}$ B. Coppi, P. Detragiache, S. Migliuolo, F. Pegoraro and F. Porcelli, Phys. Rev. Lett. 63, 2733 (1989). 